\title{
Réduire l'usage des pesticides. Un défi pour le conseil aux agriculteurs
}

The reduction of pesticides use: a challenge for extension services

\section{Philippe Aujas, Anne Lacroix, Stéphane Lemarié et Raymond Reau}

\section{(2) OpenEdition}

1 Journals

\section{Édition électronique}

URL : http://journals.openedition.org/economierurale/3071

DOI : 10.4000/economierurale.3071

ISSN : 2105-2581

\section{Éditeur}

Société Française d'Économie Rurale (SFER)

\section{Édition imprimée}

Date de publication : 30 juillet 2011

Pagination : 18-33

ISSN : 0013-0559

\section{Référence électronique}

Philippe Aujas, Anne Lacroix, Stéphane Lemarié et Raymond Reau, « Réduire l'usage des pesticides. Un défı pour le conseil aux agriculteurs », Économie rurale [En ligne], 324 | juillet-août 2011, mis en ligne le 30 juillet 2013, consulté le 10 décembre 2020. URL : http://journals.openedition.org/ economierurale/3071; DOI : https://doi.org/10.4000/economierurale.3071 


\section{Réduire l'usage des pesticides Un défi pour le conseil aux agriculteurs}

Philippe AUJAS • Direction départementale des territoires (DDT) de I'Isère, Grenoble

Anne LACROIX, Stéphane LEMARIÉ • INRA, Université Pierre Mendès France, Laboratoire d'économie appliquée de Grenoble (GAEL), Grenoble

Raymond REAU •INRA, AgroParisTech, Agronomie, Thiverval-Grignon

\section{Introduction}

a France est l'un des principaux consom$\square_{\text {mateurs de pesticides au monde. Depuis }}$ une dizaine d'années, les dommages environnementaux induits par leur usage (contamination des milieux, dégradation des écosystèmes...) sont mieux connus et différentes initiatives ont été prises pour en réduire l'utilisation. Les procédures d'autorisation de nouveaux produits sont devenues plus strictes, conduisant à une réduction importante du nombre de molécules vendues. Une taxe (TGAP) a été introduite en 2000 sur les livraisons de produits antiparasitaires à usage agricole. Enfin, différents systèmes de certification ont été mis en place pour mieux valoriser les produits agricoles issus de pratiques économes en intrants chimiques. En dehors de ces initiatives, le contexte général de baisse des prix agricoles jusqu'en 2006 a été favorable à une modération des dépenses des agriculteurs en intrants. Malgré tous ces éléments, la réduction de la consommation de pesticides est restée modeste.

Les données de l'Union de l'industrie de la protection des plantes (UIPP) montrent que les quantités de substances actives phytosanitaires vendues en France baissent au début des années 2000 (jusqu'en 2003), puis stagnent au cours des dernières années. Cette baisse globale s'explique en grande partie par une diminution des doses utilisées par les agriculteurs du fait de l'introduction de nouvelles molécules plus efficaces, mais aussi par le choix des agriculteurs de traiter à des doses inférieures aux dosages homologués. Toutefois, cet usage plus raisonné des doses de traitement s'est accompagné d'une augmentation du nombre de passages. Au final, l'Indicateur de fréquence de traitement (IFT) (Champaux, 2006) a augmenté pour certaines cultures, comme le colza pour lequel il est passé de 4,9 en 1994 à 5,1 en 2001 et 6,1 en 2006 .

Avec le Grenelle de l'environnement, le Gouvernement français a néanmoins pris des engagements forts pour l'avenir : réduire de moitié la consommation de pesticides d'ici 2018. Comme le montrent Butault et al. (op. cit.), un tel objectif ne peut être atteint par la seule diffusion de l'agriculture raisonnée et nécessite le basculement d'une partie importante des surfaces vers l'agriculture intégrée ( $c f$. encadré 1 pour plus de détails sur les notions d'agronomie). L'ambition est importante pour deux raisons principales. En premier lieu, le passage à l'agriculture intégrée nécessite un changement profond de l'ensemble des pratiques (travail du sol, semis, traitements, apport azoté, etc.) avec pour objectif de diminuer la pression de pathogènes et ainsi limiter le recours aux

1. L'IFT est défini par la somme des traitements appliqués, pondérés chacun par le rapport entre la dose utilisée par hectare et la dose d'homologation (Butault et al., 2010). L'accroissement de l'IFT signifie que l'augmentation du nombre de passages domine la baisse des doses choisies par les agriculteurs. Pour le colza, les traitements s'effectuaient à $87 \%$ de la dose homologuée en 1994 et à $78 \%$ en 2001 ; mais durant cette même période, le nombre de passages a cru de 5,7 à 6,5 (Morison, 2007). Les données de 2006 sont tirées de Butault et al. (2010). 


\section{Encadré 1. Définitions}

L'agriculture, dite raisonnée, s'inscrit dans le droit fil du cahier des charges adopté par le Conseil supérieur d'orientation et de coordination de l'économie agricole et alimentaire en mai 2002. Elle relève d'un code des « bonnes pratiques agricoles » et consiste notamment à limiter les traitements systématiques et à recourir aux produits phytosanitaires uniquement quand ils se justifient du point de vue économique. Le choix d'intervenir ou non, qu'on peut qualifier de « choix tactique » se traduit globalement par un accroissement de l'efficience des produits phytosanitaires.

L'agriculture, dite intégrée, consiste à réduire la pression des bio agresseurs en jouant sur des choix plus stratégiques (par exemple : date de semis, choix de la variété, rotation, etc.) déclinés à des échelles temporelles et spatiales plus ou moins larges (Ferron, 1999). Ainsi, on distingue les itinéraires techniques intégrés qui ont pour objectif d'améliorer l'efficacité de la conduite d'une culture sur un cycle de production à l'échelle de la parcelle, des systèmes de culture intégrés qui optimisent la succession des cultures sur plusieurs années à l'échelle de l'exploitation. La rotation qui est une donnée (ou contrainte) dans un itinéraire technique intégré devient une variable de choix stratégique dans le système de culture intégré.

produits phytosanitaires ${ }^{2}$. En second lieu, la diffusion de l'agriculture intégrée est rendue difficile par le caractère idiosynchratique de cette innovation : les meilleures pratiques diffèrent selon les exploitations, car elles doivent prendre en compte les spécificités du milieu et s'y adapter.

Du fait de ces spécificités, la protection intégrée s'avère intensive en connaissances : elle consiste à substituer du travail qualifié (traitement de l'information technique, observation, comptage, management) à des inputs (pesticides) et à du travail peu qualifié (épandage des pesticides). Dans la littérature, le niveau d'éducation est systématiquement signalé comme un facteur positif pour son adoption (Fernandez-Cornejo, 1996 ; Lohr et al., 1999). Viaux et Le Monnier (1994) constatent que limiter le nombre de traitements, limiter les doses et, de manière générale, adopter de nouvelles techniques, implique que les agriculteurs consacrent

2. Réduire significativement l'usage de pesticides revient à s'attaquer à la cohérence même des systèmes de production, car ces produits sont devenus indispensables pour maîtriser les risques phytosanitaires. Comme le montrent Meynard et Savini (2003), ces risques sont accentués par le choix de pratiques intensives : risques d'adventices et de verse accentués par l'accroissement des doses d'azote, risque d'attaques de pucerons et de maladies accentués du fait de semis précoces et denses pour augmenter le rayonnement intercepté par la culture... davantage de temps au suivi des cultures et à la prise de décision, autrement dit qu'ils accroissent leur temps de travail indirect. Carpentier (1996) met en évidence le rôle primordial de l'information dans la gestion du risque phytosanitaire et c'est l'utilisation de cette information qui permet de réduire la consommation de pesticides. Selon l'auteur, la production de cette information nécessite trois intrants : du matériel (pièges, capteurs météorologiques, logiciels de prévisions...), du temps de travail (surveillance des parcelles, analyses des données recueillies) et du capital humain (compétences). En déchargeant les agriculteurs d'une partie des tâches à effectuer pour la production de cette information, le conseil technique peut jouer un rôle important dans l'adoption de pratiques économes en pesticides (Paillotin, 2008) ${ }^{3}$.

L'objectif de cet article est d'examiner si le conseil aux agriculteurs, tel qu'il est pratiqué en France, est apte à diffuser une agriculture économe en intrants et à relever le défi du Grenelle de l'environnement. Dans quelle mesure les pratiques intégrées font-elles l'objet d'un conseil en France ?

\footnotetext{
3. Ce point se retrouve en particulier dans deux des sept axes (axes 2 et 4) mis en avant par le Comité d'orientation d'Ecophyto 2018.
} 
Sous quelle forme? Avec quels outils ? Quelle en est la fiabilité perçue par les acteurs de terrain?

La littérature met systématiquement en évidence le rôle majeur que joue le conseil dans le développement agricole et la diffusion des innovations. Néanmoins, cette activité est généralement étudiée dans son ensemble, sans se focaliser, comme nous le faisons ici, sur le conseil phytosanitaire dans le contexte particulier de réduction des intrants. Un premier groupe de travaux s'est intéressé à l'évolution de la forme institutionnelle du conseil. En sociologie, il s'agit en particulier d'analyser l'évolution du métier de conseiller (Rémy, 2006). En économie, l'analyse a porté sur les logiques de réorganisation selon les pays (Laurent et al., 2006) ou sur les incitations économiques à privatiser cette activité (Frisvold et al., 2001 ; Hanson, Just, 2001 ; Dinar, Keynan, 2001). Un second groupe de travaux s'est intéressé à la relation entre conseiller et agriculteur: d'un côté, des analyses réalisées par des sociologues et des ergonomes ont porté sur l'apprentissage de cette relation et sur la notion de co-production du conseil (Cerf, Maxime, 2006) ; de l'autre, des analyses économiques ont ciblé l'efficacité des différentes formes de conseil (visite de champs, relations bilatérales... (RickerGilbert et al., 2008 ; Godtland et al., 2004).

Notre analyse s'appuie sur le cas de la culture du colza en France. Cette culture est grosse consommatrice de produits phytosanitaires et son extension, du fait des débouchés ouverts par les biocarburants, devrait encore accroître son poids dans la consommation française de pesticides. Nous nous appuyons, entre autres, sur une enquête réalisée en 2006, auprès de 21 acteurs du système d'information et de conseil agricole, répartis dans deux régions : Bourgogne et Poitou-Charentes (encadré 2).

Dans la première section de cet article, nous cherchons à cerner et à caractériser l'organisation du conseil aux agriculteurs en France. Les deux sections suivantes explorent successivement les logiques dans lesquelles travaillent les différents acteurs du conseil et les outils d'aide à la décision dont ils disposent. Enfin, la discussion esquisse les évolutions à envisager au sein du système d'information et de conseil pour réussir le pari d'une réduction conséquente des pesticides.

\section{L'organisation du conseil agricole}

Cette première partie dresse un panorama très rapide de l'organisation du conseil en France en faisant ressortir ses spécificités dans le domaine phytosanitaire. Nous mettons l'accent en particulier sur le type de structure (publique versus privée) impliqué dans le conseil et les principales activités réalisées par ces structures.

En agriculture, la production des connaissances et le conseil se caractérisent par une forte intervention du service public. Un tel constat est cohérent avec l'explication d'Hanson et Just (op. cit.) selon laquelle le marché du conseil présente certaines défaillances qui tiennent à la nature des informations produites. En effet, une bonne partie de celles-ci ont des caractéristiques de bien public et une entreprise qui les produirait ne pourrait pas en tirer profit. En outre, la qualité de ces informations n'est guère vérifiable si bien qu'elles doivent être produites par une institution dont la réputation est solidement établie. Aussi, le secteur privé ne peut occuper que des niches où le service offert est rival et exclusif, c'est-à-dire certaines activités de front office $e^{4}$ qui correspondent à un conseil

4. Pour décrire le conseil agricole, Labarthe (2006) retient deux types de tâches : back office et front office. Les tâches de back office renvoient aux activités de recherche et développement ; elles débouchent sur la production de supports techniques : documents, outils d'aide à la décision, avertissements, essais... Les activités de front office correspondent à une relation de service entre un conseiller et un agriculteur (visites, appels téléphoniques...) ; elles permettent de contextualiser le conseil et de trouver des solutions pour gérer et adapter le système de production. 


\section{Encadré 2. Protocole d'enquête}

Les deux régions retenues illustrent deux contextes de culture du colza : le Poitou-Charentes où cette culture a été introduite récemment, la Bourgogne où elle est traditionnelle.

Un ensemble de 21 acteurs du système d'information et de conseil agricole a été choisi pour rendre compte de la diversité des organismes impliqués dans le conseil aux agriculteurs. L'échantillon compte 5 agents d'organismes d'information et de formation (service de la Protection des Végétaux, CETIOM, lycée agricole), 3 techniciens de coopératives et 3 de négoces privés, 7 conseillers de Chambre d'agriculture et 3 de cabinets de conseil indépendants.

Cette enquête réalisée pendant l'été 2006 a consisté en un entretien semi-directif. Cette méthode a l'avantage de laisser l'interviewé libre de ses propos, d'induire le moins possible les réponses et donc, dans la mesure du possible, d'éviter des réponses stéréotypées. Il nous paraissait effectivement important de ne pas enfermer les acteurs interrogés dans un positionnement visà-vis des innovations ou des formes d'agriculture : raisonnée, intégrée, d'autant que ces dernières pouvaient être définies de manière très variables d'une personne à l'autre. Aussi, seule une grille de thèmes à aborder avait été définie $: i$ ) les formes de conseil proposées en matière de conduite de culture et d'usage des pesticides (notamment les variables prises en compte dans le raisonnement et les outils utilisés), ii) les opportunités de développement de systèmes économes en produits phytosanitaires, iii) les éventuelles modifications à apporter aux formes de conseil proposées, et $i v$ ) les préconisations ou le recours aux outils d'aide à la décision.

Les interviews ont été transcrites, puis leur contenu a été analysé. Pour chaque interviewé, nous avons identifié dans son discours, les différentes pratiques économes en pesticides conseillées et sa manière de les argumenter. Cette analyse a permis d'établir différentes « logiques de conseil » en matière phytosanitaire. Dans le corps du texte, les phrases entre guillemets correspondent aux propos mot à mot des agents enquêtés. L'anonymat des personnes est respecté, mais l'appartenance institutionnelle de l'agent est systématiquement signalée. Elle est mentionnée de la manière suivante : A pour les organismes d'information et de formation, $\mathrm{C}$ pour les organismes d'approvisionnement et de collecte (coopératives et négoces), D pour les Chambres d'agriculture, B pour les cabinets de conseil indépendants. L'agent proprement dit est signalé par un numéro allant de 1 à $n$.

En outre, le matériau recueilli nous a permis de confronter l'usage qui est fait des outils d'aide à la décision avec les résultats d'une recherche bibliographique menée ex-ante [Aubertot et al. (2005) et presse professionnelle]. Cette recherche ex-ante avait pour but de faire l'inventaire des Outils d'aide à la décision (OAD) disponibles en matière de protection phytosanitaire du colza, de cerner les modalités de leur conception (notamment les connaissances qu'ils cristallisent) et leurs objectifs.

délivré directement à un agriculteur et qui, de ce fait, est contextualisé.

En France, comme dans la plupart des pays européens, le système d'information et de conseil est dominé par le secteur public, tout au moins jusqu'au milieu des années 1980. Labarthe (op. cit.) met en évidence une conception linéaire de la production des connaissances : des innovations technologiques sont induites par la recherche fondamentale, puis testées par les instituts de recherche appliquée via des expérimentations au champ et, finalement adaptées aux différentes exploitations par les services de conseil technique. Selon Laurent et al. (2006), ce système a connu de profonds changements dans les années 1990: une tendance à la privatisation/commercialisation des services de conseil, un élargissement des thèmes abordés et une réorganisation via l'utilisation des nouvelles technologies d'information et de communication. En 2004, presque la moitié des agents du conseil technique et économique aux agriculteurs est financée sur prestations payantes ou intégrées au produit (tableau 1). 
Tableau 1. Nombre d'agents (en équivalent plein-temps) de conseil et de recherche-développement

\begin{tabular}{l|c|c|c}
\hline & Nombre total & $\begin{array}{c}\text { sur financement } \\
\text { public ou } \\
\text { professionnel } \\
\text { mutualisé }\end{array}$ & $\begin{array}{c}\text { sur prestations } \\
\text { payantes } \\
\text { ou intégrées } \\
\text { au produit }\end{array}$ \\
\hline Échelon national: & 1120 & 900 & 220 \\
- instituts et centres techniques & 180 & 150 & 30 \\
- autres organismes nationaux & 6100 & 4500 & 1600 \\
\hline Échelon territorial: & 1500 & 150 & 1350 \\
- chambres d'agriculture & 1800 & 100 & 1700 \\
- coopération & 1300 & 10 & 1200 \\
- contrôles de performances & 500 & 400 & 100 \\
- centres de gestion & 300 & 300 & 100 \\
- ADASEA & 800 & 700 & 6300 \\
- syndicats & 13600 & 7300 & \\
- autres & & & \\
\hline Total & & & \\
\hline
\end{tabular}

Source : Vedel, 2006 (estimation 2004 d'après ANDA/ADAR, APCA, CFPA)

En matière de protection phytosanitaire, le recours aux conseillers est fréquent. En effet, on constate une réelle difficulté pour les agriculteurs à suivre les évolutions techniques et réglementaires dans le domaine des produits phytosanitaires. Souvent, les agriculteurs déclarent très mal connaître les produits et considèrent que l'usage de ceuxci est très compliqué. La réglementation sur les mélanges de produits phytosanitaires (arrêté du 13 mars 2006) accroît encore cet état de fait. Cela amène parfois les agriculteurs à préférer une externalisation de ce volet de la production, c'est-à-dire s'en remettre à des tiers extérieurs (techniciens phytosanitaires, conseillers de Chambres, experts privés, autres agriculteurs... ) pour choisir, décider, voire, appliquer les produits.

En France, l'offre de conseil phytosanitaire provient majoritairement d'acteurs privés, en accompagnement de la vente de produits. Aubertot et al. (op. cit.) estiment que, dans les systèmes de grandes cultures, la fréquence de contact des agriculteurs par les conseillers-vendeurs (proposant un conseil intégré au produit vendu) est 10 fois plus importante que celle enregistrée pour les conseillers stricto sensu (ceux dont le métier est uniquement le conseil) ; dans les systèmes d'élevage, ce rapport est de 25 pour 1. Cependant, en France, l'offre de ce conseil stricto sensu est beaucoup moins le fait d'entreprises privées que dans d'autres pays européens, tels que le Royaume-Uni, l'Allemagne, les Pays-Bas (Labarthe, op. cit.). L'implication de structures publiques est donc importante et permet une offre diversifiée. Ainsi, depuis le début des années 80, les Chambres d'agriculture ont à la fois accru leurs services individuels, réduit leur offre purement technique pour s'ouvrir à des domaines plus novateurs, tels que la gestion de l'environnement (Mundler et al., 2006).

Au total, si on adopte une définition large du conseil qui recouvre les activités de recherche-développement jusqu' aux services à l'entreprise, force est de constater la multiplicité et la variété des fournisseurs de conseil agricole en France. La question se pose alors de savoir si cette diversité de structure conduit à une pluralité de type de conseil délivré. Plus précisément, il s'agit de cerner la place qui est donnée à la mise en œuvre de pratiques économes en produits phytosanitaires par les principales catégories d'acteurs répertoriées : organismes d'information et de formation, coopératives, négoces privés, Chambres d'agriculture, cabinets de conseil indépendants. L'usage des pesticides s'avère un bon angle d'observation des pratiques de conseil dans la 
mesure où il s'agit d'un domaine très technique que les agriculteurs délèguent volontiers aux conseillers et que les réponses apportées pour réduire les pesticides peuvent relever de l'agriculture raisonnée ou de l'agriculture intégrée (cf. encadré 1).

\section{Les différentes logiques de conseil}

$\mathrm{Au}$ cours de notre enquête, plusieurs interlocuteurs ont relevé un intérêt marqué de la part des agriculteurs pour des conduites économes en produits phytosanitaires. Les raisons évoquées sont avant tout économiques, en lien avec le « ciseau des prix » : en grande culture, la baisse des prix des produits se conjuguait alors (été 2006) à une hausse des prix des matières premières et des équipements. La réponse faite à cette demande par les acteurs enquêtés n'est pas uniforme : toute une palette de pratiques moins intensives existe et nous verrons qu'elle est rarement proposée dans son ensemble par les conseillers.

Ainsi, lors de notre enquête, nous avons noté que les agents technico-commerciaux des Organismes d'approvisionnement et de collecte (OAC) donnent la priorité aux techniques permettant d'obtenir les meilleurs rendements. Viser des rendements élevés est souvent affiché comme le seul moyen pour les agriculteurs de faire les meilleures marges. Et le risque de perdre des quintaux est mis en avant pour expliquer la non-réduction de l'usage des produits phytosanitaires : "En 1992, l'arrêt des traitements durant deux à trois ans a entraîné une baisse des rendements. » $(\mathrm{C} 3)^{5}$. Entre deux alternatives de lutte contre les adventices, la priorité est donnée à la technique la moins coûteuse à court terme et c'est ce qui justifie la préférence pour la lutte chimique : «Le chimique est moins cher que le mécanique. » (C1).

$\mathrm{Ce}$ raisonnement, très fréquent chez les conseillers-vendeurs, est également présent

$\overline{\text { 5. Cf. l'encadré p. } 21}$ : l'identité de l'agent conseil. chez certains agents des Chambres d'agriculture qui restent très attachés à la valeur « rendement », et peu ouverts aux conduites économes : "On ne peut pas cultiver $d u$ colza avec des intrants au rabais. "(D4). L'argument avancé ici n'est pas l'argument économique, mais plutôt le risque que prend le conseiller d'être réprimandé en cas de rendement déficient : "Un agriculteur en voudra davantage à son conseiller s'il lui fait perdre des quintaux que s'il lui fait faire une dépense de traitement inutile, car il ne voit pas forcément que le traitement est inutile. » (D4).

Cet objectif de rendement élevé conduit les conseillers à maintenir leurs préconisations habituelles, mais en ayant le souci de respecter les nouvelles obligations réglementaires. Désormais, leur conseil intègre un rappel des bonnes pratiques à respecter et la mise à disposition d'outils pour aider les agriculteurs à justifier leurs traitements phytosanitaires. Il s'agit de leur faciliter la tâche, en cas de contrôle, en leur permettant de conserver la trace de leurs pratiques culturales.

Cependant, d'autres techniciens de Chambre d'agriculture et les agents exerçant au sein de cabinets indépendants affichent clairement l'objectif d'accompagner les agriculteurs dans leur recherche de réduction de charges. "Dans les années 1970, le conseil était purement technique. À partir de 1992, l'orientation vers l'optimisation des intrants a été prise. Aujourd'hui, l'objectif est de créer de la valeur ajoutée... L'agriculture intégrée, correspond à une proposition de solution. » (D5) «La réduction des charges est un moyen de maintenir, voire d'augmenter la marge. »(D1). Cependant, l'attente des agriculteurs semble varier avec le potentiel de leurs terres; elle serait d'autant plus pressante que leurs terres sont de qualité médiocre : "Les agriculteurs sont attentifs aux essais visant à réduire les charges, car les rendements blé sur le secteur sont modérés: 50-60 quintaux. » (D1). 
Pour réduire les charges, notamment les dépenses de pesticides, différentes solutions sont préconisées.

- La première, qui est surtout le fait des conseillers des OAC, consiste à adapter la nature du produit phytosanitaire au niveau de risque : un produit bon marché si le risque est faible, un produit onéreux sinon. "Le sclérotinia n'est pas facile à raisonner. On est davantage dans le systématique... modulé suivant le climat : une carbendazime si le risque climatique est faible; un produit haut de gamme en cas de risque fort » $(\mathrm{C} 4)$.

- La deuxième solution, recommandée par les OAC, mais aussi par certains instituts techniques, implique le recours à de nouvelles variétés plus résistantes aux maladies. Cette stratégie est mise en avant tout particulièrement pour le colza, du fait de l'arrivée des hybrides sur le marché 6 . Du choix de variété découlerait nombre de décisions culturales : "Le mode d'emploi de l'itinéraire technique, c'est la variété qui le détermine. " (C2). "Le conseil se fait [...] à la variété et non pas à la parcelle » (C4).

- Une troisième solution, pour réduire les charges vise explicitement une diminution des pesticides. Elle est mise en avant par un agent du service de la protection des végétaux, mais aussi par un agent d'un institut technique. Il s'agit d'augmenter le seuil de bio agresseurs à partir duquel il est conseillé de traiter ou d'agir sur leur population en implantant des plantes pièges, comme par exemple les mélanges variétaux ou les bandes fleuries pour piéger les méligèthes.

6. L'usage de semences hybrides contraint l'agriculteur à acheter sa semence tous les ans. Du point de vue de l'OAC, la baisse des ventes de produits phytosanitaires ne se solde pas forcément par une baisse du chiffre d'affaires, car elle est en partie compensée par des ventes de semences plus fréquentes.
La stratégie plus novatrice qui consiste à changer l'itinéraire technique de la culture en vue de diminuer la pression ou les dégâts des bio agresseurs est testée par certains agents de Chambre d'agriculture, par des cabinets de conseil indépendants et par un lycée ; mais, elle reste en débat. Les uns s'appuient sur les résultats du réseau «blé rustique ${ }^{7}$ pour affirmer l'intérêt des conduites économes : "Les protocoles sur le blé rustique reposent sur la baisse d'intrants pour des raisons économiques. La réduction d'intrants concerne les fongicides, les régulateurs de croissance, la fumure azotée, la densité de semis. Les protocoles se font sur des pratiques simples à réaliser sur différentes variétés. Une dizaine d'essais ont été réalisés jusqu'ici. Résultats : en 2005, il existe un écart de 2 quintaux en faveur des conduites classiques, mais la marge brute est meilleure sur le blé rustique pour une qualité égale» (D3). D'autres restent sceptiques : l'avantage donné au blé rustique serait biaisé car "les itinéraires techniques de référence sont trop intensifs et le choix des produits porte sur des produits chers. Cela favoriserait les résultats en faveur de la conduite intégrée. »(D1). Des doutes existent aussi sur la possibilité d'aboutir à de telles performances pour le colza : "L'expérience du blé rustique est difficile à reproduire sur le colza car le principal problème du colza, le désherbage, est lié aux rotations courtes sur le secteur. »(D3). Pour la culture du colza, le changement d'itinéraire technique serait délicat : "Sur le colza, on peut travailler sur certains points, comme la fertilisation azotée ou la date de semis. Mais [...] le matériel n'est pas forcé-

7. Ce réseau conduit des expérimentations d'itinéraires techniques intégrés en blé. En combinant le choix de variétés résistantes aux maladies, une réduction de la densité de semis (-40\%), de la dose d'azote $(-20 \%)$, des traitements fongicides $(-70 \%)$ et une suppression des régulateurs de croissance, les rendements sont réduits de 10 à $15 \%$ et les marges brutes sont au moins équivalentes à celles des itinéraires techniques conventionnels, sans que la variabilité des résultats soit accrue (Loyce et al., 2001). 
ment adapté à la réduction de densité. Une impasse fongicide sur le sclérotinia peut être préjudiciable au rendement et constitue un risque de réensemencement des sclérotes dans le sol. L'impasse sur le désherbage est une aberration; le binage laisse les adventices sur le rang. »(D1).

Parmi les enquêtés, quelques rares agents du développement envisagent des solutions de plus long terme pour réduire les pesticides : changement de rotation pour maîtriser la pression des adventices ; modification de l'ensemble du système de culture pour réduire la pression de tous les bio agresseurs. Le changement de rotation prend toute son importance dans le cas du colza, car certains adventices s'avèrent très difficiles à maîtriser : "L'entrée prioritaire, c'est donc la rotation, notamment pour le désherbage. "(D2). Cependant, le choix des rotations est souvent considéré comme le résultat des besoins du marché : «Le choix de la rotation résulte d'un compromis entre l'économique et l'agronomique. » (C5). Le changement de système de cultures n'a été abordé qu'une seule fois, dans une Chambre d'agriculture : "C'est sur l'ensemble du système que l'on doit estimer le risque [...] le système intégré doit se mettre progressivement en place au niveau de l'exploitation. »(D2). Cette problématique est rarement abordée dans les OAC : « $L a$ coopérative ne peut pas conseiller sur des itinéraires incertains et s'investir sur des essais à long terme. L'agriculteur souhaite des solutions immédiates et rentables à court terme : un tiers des agriculteurs sera à la retraite dans cinq à six ans. "(C5).

En résumé, tous les acteurs du conseil rencontrés lors de l'enquête s'accordent sur la nécessité de raisonner l'usage des phytosanitaires, plutôt que procéder à des traitements systématiques. Cependant, les solutions qu'ils préconisent pour ce faire sont plus ou moins radicales et le récapitulatif en est donné dans le tableau 2. Pour les uns, notamment les conseillers-vendeurs, ceci se limite à cibler la date d'application du produit, adapter sa nature, voire sa dose, afin de pouvoir justifier de «bonnes pratiques ». Pour les autres, il s'agit de réduire la fréquence de traitement en changeant la variété, en modifiant les seuils d'intervention... Enfin, une minorité propose des solutions plus globales en jouant sur l'itinéraire technique, la rotation ou le système de cultures. Notre enquête montre que les solutions proposées pour limiter les pesticides relèvent d'un spectre plus large que l'opposition agriculture raisonnée/agriculture intégrée. Cependant, elle met en évidence que peu de conseillers, parmi les interviewés, investissent des solutions relevant de la production intégrée. Deux raisons principales peuvent être avancées pour expliquer cet état de fait. En premier lieu, il est difficile d'infléchir les décisions de nombreux agriculteurs compte tenu du prix modéré des pesticides par rapport aux pertes potentielles de rendement en valeurs. En second lieu, délivrer un conseil efficace en faveur des itinéraires techniques intégrés

Tableau 2. Récapitulatif des solutions préconisées selon le type de structure de conseil

\begin{tabular}{l|c|c|c|c}
\hline & $\begin{array}{c}\text { Organismes } \\
d^{\prime} \text { information et } \\
\text { de formation }\end{array}$ & $\begin{array}{c}\text { Organismes } \\
\text { Approvisionnement } \\
\text { et Collecte }\end{array}$ & $\begin{array}{c}\text { Chambres } \\
\text { d'agriculture }\end{array}$ & $\begin{array}{c}\text { Cabinets } \\
\text { de conseil } \\
\text { indépendants }\end{array}$ \\
\hline Optimiser l'usage des pesticides & $\mathrm{A} 2, \mathrm{~A} 3, \underline{\mathrm{A} 4}$ & $\begin{array}{c}\mathrm{C} 1, \mathrm{C} 2, \underline{\mathrm{C}} ; \mathbf{C} 4 ; \\
\mathrm{C} 5, \mathrm{C} 6\end{array}$ & $\underline{\mathrm{D} 1, \mathrm{D} 3, \underline{\mathrm{D} 4}, \mathrm{D} 5}$ & $\mathrm{~B} 1, \underline{\mathrm{B} 2}$ \\
\hline $\begin{array}{l}\text { Choisir une variété réduisant } \\
\text { les risques sanitaires }\end{array}$ & $\mathrm{A} 3, \underline{\mathrm{A} 4}$ & $\mathrm{C} 2, \underline{\mathrm{C} 3} ; \underline{\mathrm{C} 4} ;$ & $\mathrm{D} 2, \underline{\mathrm{D} 3}, \mathrm{D} 5$, & $\mathrm{B} 1, \underline{\mathrm{B} 3}$ \\
\hline $\begin{array}{l}\text { Gérer la rotation pour limiter } \\
\text { la pression des bio agresseurs }\end{array}$ & $\mathrm{A} 1, \mathrm{~A} 5$ & & $\mathrm{D} 2, \mathrm{D} 6, \mathrm{D} 7$ & \\
\hline
\end{tabular}

Note : Les structures de conseil situées en Poitou-Charentes sont soulignées. 
est complexe, car il doit couvrir un ensemble de décisions tout en étant adapté aux spécificités de chaque exploitation. Néanmoins, différents outils d'aide à la décision ont été développés pour faciliter ce conseil. Nous allons à présent étudier leur contenu et l'usage qui en est fait pour apprécier leur capacité à diffuser une démarche de production intégrée.

\section{Les outils de raisonnement de l'usage des pesticides}

Les OAD qui servent de support au conseil, cristallisent les connaissances acquises via la recherche-développement. Ils ont connu un développement notable au début des années 2000 du fait de l'émergence des nouvelles technologies d'information et de communication. En matière de protection sanitaire, notre recherche bibliographique nous a permis de mettre en évidence quatre types d'OAD, différenciés selon les procédures de collecte de l'information qu'ils utilisent et donc sur la plus ou moins grande généricité du conseil qui en résulte :

- les modèles de prévision de l'évolution des maladies ou des populations d'insectes en fonction des conditions climatiques (température, pluviométrie, hygrométrie);

- les bulletins d'avertissements agricoles basés sur les résultats des modèles, des suivis de parcelles de référence et des informations émanant des différents réseaux de techniciens ;

- les documents techniques édités sous différents formats (plaquettes, bases de données, logiciels) ;

- les kits de diagnostic.

Pour l'ensemble des grandes cultures, les outils proposés sont nombreux ; concernant le colza seul, la liste est beaucoup plus limitée (tableau 3). Cette liste se restreint si on ne retient que les outils réellement utilisés par les agents du conseil. En outre, comme le soulignent Cerf et Meynard (2006), il existe parfois un écart important entre l'usage prévu lors de la conception même de l'outil et l'usage qui en fait par les conseillers ou les agriculteurs.

Le Service de protection des végétaux (SPV) a développé des modèles épidémiologiques afin de prévoir l'arrivée des bio agresseurs et procéder à des avertissements régionaux. Ces outils ont, le plus souvent, été bâtis dans l'optique d'assurer l'efficacité des traitements, en essayant d'identifier le moment le plus opportun pour les réaliser. Cependant, ces modèles n'ont pas toujours été à la hauteur des attentes : "Au départ, l'attente était forte vis-à-vis des modèles mais cela n'a pas révolutionné la préconisation. Le modèle est trop réducteur et touche vite ses limites. »(A2). Leur sensibilité semble notamment en cause : "Le modèle phoma du Service de la protection des végétaux, on ne sait pas s'il existe ou pas. Chaque année c'est le même conseil! »(B3).

Les avertissements du SPV sont très souvent utilisés par les coopératives et négoces : "L'avertissement de la Protection des végétaux est affiché dans les dépôts. Le service technique peut moduler la préconisation de la Protection de végétaux en fonction du contexte local. Je déclenche le traitement par un "top traitement»: date, produit, dose. »(C3). Dans les organismes d'approvisionnement et de collecte, cet outil est donc utilisé pour raisonner les traitements phytosanitaires, mais aussi pour les justifier dans le cadre des cahiers des charges, tels que celui de l'agriculture raisonnée, ou lors d'éventuels contrôles sur l'éco-conditionnalité. "L'éco-conditionnalité, la réglementation phytosanitaire sont évoquées lors des réunions [...] Ceci favorise la demande d'outils d'aide à la décision pour la fertilisation azotée et la protection phytosanitaire. »(C5). Ces mêmes organismes ont d'ailleurs mis au point un registre phytosanitaire qui permet d'assurer la traçabilité des pratiques à cet effet. 
RECHERCHES

Philippe AUJAS, Anne LACROIX, Stéphane LEMARIÉ, Raymond REAU

Tableau 3. Les principaux types d'OAD disponibles pour la culture de colza

\begin{tabular}{|c|c|c|c|c|}
\hline Type d'outil & Information recueillie & $\begin{array}{c}\text { Traitement } \\
\text { de l'information }\end{array}$ & $\begin{array}{l}\text { Type de décision } \\
\text { éclairée }\end{array}$ & $\begin{array}{l}\text { Exemples pour } \\
\text { la culture du colza }\end{array}$ \\
\hline $\begin{array}{l}\text { Grille de risques } \\
\text { (maladie) }\end{array}$ & $\begin{array}{l}\text { Caractéristiques de la } \\
\text { parcelle : sol, } \\
\text { pratiques culturales } \\
\text { essentielles, climat }\end{array}$ & $\begin{array}{l}\text { Modèles factoriels } \\
\text { additifs, avec score }\end{array}$ & $\begin{array}{l}\text { Opportunité du } \\
\text { traitement fongicide }\end{array}$ & $\begin{array}{l}\text { Grille « sclérotinia » } \\
\text { Grille « phoma » }\end{array}$ \\
\hline $\begin{array}{l}\text { Modèle } \\
\text { épidémiologique } \\
\text { pour avertissement } \\
\text { agricole }\end{array}$ & $\begin{array}{l}\text { Caractéristiques } \\
\text { de la parcelle } \\
\text { Climat récent }\end{array}$ & $\begin{array}{l}\text { Modèle } \\
\text { épidémiologique }\end{array}$ & $\begin{array}{l}\text { Date d'arrivée } \\
\text { prévisible des } \\
\text { ravageurs, de la } \\
\text { maladie } \\
\text { Positionnement du } \\
\text { traitement } \\
\text { phytosanitaire }\end{array}$ & $\begin{array}{l}\text { Modèle « Proplant } \\
\text { ravageurs colza » } \\
\text { Modèle « Positif » } \\
\text { Modèle « Sclérotinia » } \\
\text { (SRPV) }\end{array}$ \\
\hline $\begin{array}{l}\text { Kit de diagnostic } \\
\text { (insectes) au champ }\end{array}$ & $\begin{array}{l}\text { Présence et impor- } \\
\text { tance des insectes } \\
\text { présents dans un } \\
\text { piège normalisé }\end{array}$ & $\begin{array}{l}\text { Comptage et } \\
\text { évaluation par } \\
\text { rapport à un seuil }\end{array}$ & $\begin{array}{l}\text { Connaissance de la } \\
\text { dynamique d'exten- } \\
\text { sion du ravageur }\end{array}$ & $\begin{array}{l}\text { Cuvette jaune (piège } \\
\text { à insectes) }\end{array}$ \\
\hline $\begin{array}{l}\text { Adaptation des } \\
\text { techniques culturales } \\
\text { entre elles }\end{array}$ & $\begin{array}{l}\text { Pratiques culturales } \\
\text { déjà réalisées, ou } \\
\text { programmées }\end{array}$ & $\begin{array}{l}\text { Adaptation de la } \\
\text { lutte chimique, ou de } \\
\text { la variété en fonction } \\
\text { des autres moyens } \\
\text { de lutte }\end{array}$ & $\begin{array}{l}\text { Opportunité du } \\
\text { traitement en } \\
\text { fonction de la variété } \\
\text { ou choix de la variété } \\
\text { suivant les variétés } \\
\text { semées par le passé }\end{array}$ & $\begin{array}{l}\text { Choix des alternances } \\
\text { variétales (phoma) }\end{array}$ \\
\hline $\begin{array}{l}\text { Outil tactique de } \\
\text { raisonnement } \\
\text { (fongicide) }\end{array}$ & $\begin{array}{l}\text { État sanitaire observé } \\
\text { de la parcelle (ex. : conta- } \\
\text { mination d'un organe } \\
\text { par une maladie) }\end{array}$ & $\begin{array}{l}\text { Évaluation de risque } \\
\text { à partir d'un seuil de } \\
\text { référence et d'un } \\
\text { modèle statistique }\end{array}$ & $\begin{array}{l}\text { Opportunité du } \\
\text { traitement en } \\
\text { fonction de l'état de } \\
\text { la culture observé }\end{array}$ & Kit fleur (sclérotinia) \\
\hline $\begin{array}{l}\text { Outil stratégique de } \\
\text { choix d'un program- } \\
\text { me de lutte contre } \\
\text { les adventices }\end{array}$ & $\begin{array}{l}\text { Niveau d'infestation } \\
\text { de la parcelle } \\
\text { Caractéristiques de la } \\
\text { parcelle : sol, climat }\end{array}$ & $\begin{array}{l}\text { Système expert } \\
\text { d'évaluation du } \\
\text { risque } \\
\text { malherbologique, et } \\
\text { module de choix } \\
\text { multicritère }\end{array}$ & $\begin{array}{l}\text { Choix d'un program- } \\
\text { me de lutte contre les } \\
\text { adventices dans une } \\
\text { culture (techniques, } \\
\text { économiques, risques } \\
\text { environnementaux...) }\end{array}$ & $\begin{array}{l}\text { Logiciel } \\
\text { « DECID’herb » }\end{array}$ \\
\hline $\begin{array}{l}\text { Base de données } \\
\text { d'efficacité des produits } \\
\text { phytosanitaires }\end{array}$ & & $\begin{array}{l}\text { Requêtes dans } \\
\text { une base de données } \\
\text { de résultats } \\
\text { expérimentaux } \\
\end{array}$ & Efficacité, sélectivité... & $\begin{array}{l}\text { Efficol } \\
\text { (phytosanitaires) }\end{array}$ \\
\hline $\begin{array}{l}\text { Référentiel } \\
\text { caractérisant les } \\
\text { variétés disponibles }\end{array}$ & & $\begin{array}{l}\text { Requêtes parmi les } \\
\text { connaissances } \\
\text { synthétiques dispo- } \\
\text { nibles pour chaque } \\
\text { variété }\end{array}$ & $\begin{array}{l}\text { Une synthèse des } \\
\text { caractéristiques des } \\
\text { variétés. Les variétés } \\
\text { adaptées à un } \\
\text { contexte donné }\end{array}$ & Oleov@r (variétés) \\
\hline
\end{tabular}

Source : Aubertot et al. (2005), presse professionnelle et entretiens réalisés par les auteurs. 
Pour les agents des Chambres d'agriculture impliqués dans la protection intégrée, la priorité est donnée aux outils qui permettent de raisonner localement les traitements, en évaluant le risque au niveau d'une parcelle en particulier : "Les OAD doivent être complétés par des observations. "(D5). L'utilisation des modèles devient alors complexe puisqu'il convient de les renseigner via des observations sur l'état de la parcelle. Des outils plus faciles à manier existent, tels que les kits de diagnostic au champ, mais ils ne s'avèrent pas toujours efficaces : "Le kit pétale sur colza n'est pas un outil opérationnel. »(D5).

Au total, la plupart des OAD disponibles et la quasi-totalité de ceux qui sont utilisés visent à raisonner la date ou le positionnement du traitement phytosanitaire pour garantir son efficacité. Par contre, plus rares sont les outils tactiques permettant de juger de l'opportunité de l'application d'un traitement phytosanitaire compte tenu de l'état de la parcelle. Ils sont paramétrés pour éviter tout dégât ; ceci pousse imperceptiblement à rechercher «l'excellence » tout au long du cycle de la culture. En outre, notre enquête a mis en évidence qu'ils raisonnent chaque couple culture/bio-agresseur de façon séparée. Cette pratique conduit donc généralement à mettre en avant, pour chaque facteur étudié, la modalité qui permet d'obtenir le rendement maximum. En somme, le paramétrage des OAD et leur conception cloisonnée conduisent à accroître les traitements par un système basé sur l'additivité. Or, l'itinéraire technique le plus intéressant pour limiter la pression des bio agresseurs ne résulte pas de la somme des meilleures modalités de chaque intervention, mais de la valorisation de l'interaction des différents éléments (notamment eau, sol, plante, fertilisants et pesticides) qui composent l'écosystème. Pour ce faire, il conviendrait de disposer d'outils capables de valoriser ces interactions.

Des outils spécifiquement conçus pour choisir a priori des stratégies de protection intégrée sont actuellement proposés par la recherche. En France, on peut citer le logiciel de gestion de la flore adventice à la parcelle DECID'herb développé dans le cadre d'une collaboration entre l'INRA, ARVALISInstitut du végétal et le Centre technique interprofessionnel des oléagineux métropolitains (CETIOM). Il prend en compte le risque malherbologique, l'efficacité a priori des programmes candidats et notamment de la rotation culturale, leurs coûts, leurs impacts environnementaux, ainsi que des critères techniques d'organisation du travail et de risque de sélection de populations résistantes aux herbicides. Au Royaume-Uni, la fondation Rothamsted Research développe actuellement un outil intégré, nommé DEcision Support System for Arable Crops (DESSAC). Cet outil est capable d'analyser l'impact en termes d'efficacité et de coûts de différentes pratiques culturales (dates de traitement, doses de fongicides et d'insecticides, choix de variétés...).

Malgré tout, les propositions de la recherche restent bien en deçà des outils qui seraient nécessaires pour accompagner le processus d'apprentissage de la protection intégrée par les agriculteurs et leurs conseillers. Cette défaillance tient aux difficultés de la recherche-développement pour mener une approche holistique des systèmes ; approche qui relèverait du croisement de plusieurs disciplines (Atkinson, McKinlay, 1997). Or, la France et, sans doute aussi l'ensemble des pays occidentaux, auraient délaissé ce type de recherche. Un bilan effectué en 1990 (Girardin, 1992) montrait que moins de $5 \%$ des agronomes de l'INRA travaillaient sur les « systèmes de cultures ». L'auteur expliquait ce quasi-désengagement par deux niveaux de difficultés qui se renforcent mutuellement : la difficulté personnelle des chercheurs à échapper à des stratégies de minimisation des risques intellectuels, financiers et de marginalisation ; la difficulté culturelle à concevoir des méthodes de recherche de synthèse et donc à organiser des programmes pluridisciplinaires sur le long terme. 
Plus précisément, les investigations de la R \& D en matière d'agriculture intégrée restent timides du fait des méthodologies expérimentales inappropriées. En effet, l'essentiel des expérimentations menées jusqu'alors sont factorielles, c'està-dire ont fait varier les facteurs (variété, produit de traitement, règle de décision simple...) de manière indépendante, en supposant que les interactions entre les différentes techniques culturales sont de faible importance. Du fait que la production intégrée consiste justement à valoriser ces interactions, ces essais s'avèrent insuffisants. Par contre, ils peuvent servir de base pour développer des modèles qui permettent d'éviter les coûts prohibitifs d'expérimentations multifactorielles qui testeraient toutes les interactions entre toutes les modalités de toutes les techniques culturales (Reau et al., 1996). Les résultats de modélisation servent alors à sélectionner les itinéraires techniques intéressants. Mais, en raison de l'aptitude prédictive encore limitée des modèles développés, la vérification expérimentale reste incontournable. Cette expérimentation porte alors sur les modes de conduite des cultures sélectionnés et permet d'expliciter les règles de décision de ces conduites. Elle vérifie la performance de ces combinaisons de techniques dans le moyen/long terme et pour des milieux divers.

\section{Conclusion}

Cet article étudie le conseil phytosanitaire en France en prenant l'exemple de la culture du colza. Il s'appuie sur une enquête réalisée auprès d'une vingtaine de conseillers représentatifs de la diversité des offreurs de conseil, localisés dans deux régions (Bourgogne et Poitou-Charentes) et qui, du fait de l'ancienneté de la culture de colza dans chacune de ces régions, bénéficient d'un savoir-faire et de routines plus ou moins importants. Cette étude montre que, malgré un contexte économique, une volonté politique et une réglementation propices à la réduction des pesticides, le conseil agricole est orienté en grande partie vers le raisonnement et la justification de l'application de produits phytosanitaires. En revanche, assez peu de conseils sont délivrés en faveur de stratégies plus novatrices visant à limiter la pression de bio agresseurs, telle que l'agriculture intégrée. Ce résultat tient à quatre constats principaux :

i) l'offre de conseil est largement dominée par les technico-commerciaux des coopératives et négoces dont l'activité porte également sur la vente de produits phytosanitaires (Aubertot et al., op. cit.) ;

ii) les prescriptions des conseillers de coopératives et de négoces donnent la priorité à l'efficience et à la justification des traitements plutôt qu'à la réduction de la dépendance de l'agriculture vis-à-vis des produits phytosanitaires ;

iii) les OAD disponibles ne permettent guère une approche systémique et donc sont peu adaptés pour concevoir des systèmes de culture intégrés ;

iv) les méthodologies et les connaissances accumulées jusqu'ici, la manière dont elles sont produites et partagées, limitent la capacité à développer des pratiques intégrées innovantes.

Gardons en tête néanmoins que ces résultats sont établis pour une seule culture, le colza, et à partir d'une enquête menée auprès d'un nombre restreint d'agents du conseil. Ces résultats mériteraient d'être confirmés auprès d'un échantillon plus grand, couvrant un éventail plus large de régions et de cultures. En outre, une telle enquête devrait être effectuée régulièrement afin de suivre les changements importants dans ce secteur. Malgré ces réserves méthodologiques, il nous semble que ces résultats ont une portée plus générale dans la mesure où ils convergent avec les constats de défaillance et les recommandations d'autres auteurs concernant l'organisation actuelle de la recherche et du 
développement agricole (Paillotin, op . cit.).

Ces résultats soulèvent des questions importantes quant aux politiques à mettre en œuvre pour faire évoluer le système actuel de conseil agricole. Dans cette optique, certains auteurs ont préconisé de mettre en place une politique double, combinant d'une part des mesures qui poussent au changement, comme par exemple le développement des compétences des conseillers, et d'autre part des mesures qui tirent ce changement, comme par exemple la sensibilisation des agriculteurs ou le recours à des incitations économiques (Ingram, Morris, 2007 ; Botha et al., 2008 ; Klerkx, Jansen, 2010). Nous terminerons cet article en évoquant les perspectives sur ces deux volets des politiques publiques à mettre en place et les questions qui mériteraient de plus amples recherches.

L'accumulation de connaissances dans le domaine de l'agriculture intégrée nécessite une plus grande synergie entre recherche, enseignement et pratique (Jeger, 1997). Un effort de synthèse des connaissances est indispensable. Pour ce faire, il conviendrait de rassembler les résultats issus de différents horizons (recherches disciplinaires, savoir-faire des producteurs...), de mener des expérimentations à la ferme en y impliquant les agriculteurs eux-mêmes. En outre, Lamine et al. (2009) montrent qu'un tel changement suppose des apprentissages importants, basés en particulier sur l'accompagnement par des conseillers, mais également sur les dynamiques collectives au sein de groupes d'agriculteurs. Plusieurs évolutions récentes vont dans ce sens. Les Unités et les Réseaux mixtes technologiques (UMT et RMT) constituent un premier pas décisif pour coordonner la recherche, le développement et la formation, pour définir les priorités et organiser le transfert auprès des acteurs. Par ailleurs, la constitution de réseaux régionaux dans le cadre du Système de conseil agricole (SCA) peut favoriser certains apprentissages collec- tifs. Du point de vue économique, ces dispositifs présentent certains points communs avec les dispositifs décentralisés dans lesquels l'innovation est réalisée par les utilisateurs (ex. sélection participative en amélioration des plantes, participation des utilisateurs au développement de logiciels libres). Il serait intéressant ici d'étudier dans quelle mesure ces autres expériences peuvent être transposées au cas de la recherche sur l'agriculture intégrée, et d'étudier les conditions dans lesquelles ces dispositifs décentralisés sont efficaces du point de vue économique.

Outre le fait de renforcer les connaissances et les compétences sur l'agriculture intégrée, il conviendrait aussi d'inciter les organismes de conseil agricole à modifier leurs prestations. En suivant Lucas et Meynard (2000), on peut recommander que les services à offrir ne se situent plus uniquement dans le domaine du raisonnement tactique, mais doivent s'élargir à un raisonnement stratégique mené pour la durée de la campagne, voire à plus long terme, et pour un îlot de parcelles, voire une petite région.

Pour infléchir les prestations offertes, on peut tout d'abord recourir à des instruments visant à limiter la consommation de produits phytosanitaires par les agriculteurs (comme par exemple la taxe) et favorisant ainsi la demande pour un conseil ciblé sur des pratiques moins intensives.

On peut aussi porter la réflexion sur la mise en place d'une réglementation de l'activité de conseil. Le plan Ecophyto 2018, mis en place à la suite du Grenelle de l'environnement, prévoit de rendre obligatoire la certification des structures délivrant un conseil à l'utilisation des pesticides indépendamment d'une activité de distribution.

Cette perspective n'épuise pas les questions soulevées par notre étude puisque la certification ne concerne ni les coopératives, ni les négoces. Faut-il séparer les activités de conseil de celles de vente de 
produits, ou tout au moins obliger les acteurs à facturer le service de conseil séparément de la vente de produits ? Très peu d'analyses économiques ont été réalisées sur ces questions. Au fond, le problème soulevé est celui des modalités d'organisation de l'offre d'expertise et un tel travail pourrait s'appuyer sur des travaux d'économie industrielle et en particulier procéder par analogie avec des services tels que la réparation automobile ou les prestations médicales (Dulleck, Kerschbamer 2006).
Les auteurs tiennent à remercier les 21 acteurs du conseil et développement qui ont accepté de leur consacrer un peu de leur temps précieux. Ils tiennent aussi à remercier les rapporteurs anonymes de la revue qui ont permis d'accroitre la lisibilité de l'article.

Les investigations de cet article ont été menées dans le cadre d'un projet de recherche pluridisciplinaire intitulé "Conduite intégrée du colza d'hiver pour une réduction de l'utilisation des pesticides » et financé par le ministère de I'Environnement et du Développement Durable (Programme "Évaluation et réduction des risques liés à l'utilisation des pesticides »).

\section{RÉFÉRENCES BIBLIOGRAPHIQUES}

Atkinson D., McKinlay R.-G. (1997). Crop protection and its integration within sustainable farming systems. Agriculture, Ecosystems \& Environment, vol. 64, $\mathrm{n}^{\circ} 2$, p. 87-93.

Aubertot J.-N., Barbier J.-M., Carpentier A., Gril J.-J., Guichard L., Lucas P., Savary S., Savini I., Voltz M. (éds.) (2005). Pesticides, agriculture et environnement. Réduire l'utilisation des pesticides et limiter leurs impacts environnementaux. Rapport d'Expertise scientifique collective, INRA et Cemagref.

Botha N., Coutts J., Roth H. (2008). The role of agricultural consultants in New Zealand in environmental extension. The Journal of Agricultural Education and Extension, $\mathrm{n}^{\circ} 14$, p. 125-138.

Butault J.-P., Dedryver C.-A., Gary C., Guichard L., Jacquet F., Meynard J.-M., Nicot P., Pitrat M., Reau R., Sauphanor B., Savini I, Volay T., Ecophyto R \& D (2010). Quelles voies pour réduire l'usage des pesticides? Synthèse du rapport d'étude, INRA Éditeur France, $90 \mathrm{p}$.

Carpentier A. (1996). Efficacité privée et publique de la gestion du risque phytosanitaire : le rôle de l'information. Cahiers d'Économie et Sociologie Rurales, $\mathrm{n}^{\circ} 39$ 40, p. 37-61.
Cerf M., Meynard J.-M. (2006). Les outils de pilotage des cultures : diversité de leurs usages et enseignements pour leur conception. Natures, Sciences, Sociétés, ${ }^{\circ} 14$, p. 19-29.

Cerf M., Maxime F. (2006). La coproduction du conseil : un apprentissage difficile. In Rémy J., Brives H., Lémery B. (éds.), « Conseiller en agriculture », coédition Éducagri-INRA, p. 137-152.

Champeaux C. (2006). Dépendance des grandes cultures à l'utilisation de pesticides. Évolution de l'IFT au travers des enquêtes Pratiques Culturales du SCEES entre 1994 et 2001. INRA Grignon/ ministère de l'Agriculture et de la Pêche, $54 \mathrm{p}$.

Dinar A., Keynan G. (2001). Economics of paid extension: lessons from experience in Nicaragua. American Journal of Agricultural Economics, n ${ }^{\circ}$ 83, p. 769-776. Dulleck U., Kerschbamer R. (2006). On doctors, mechanics, and computer specialists: the economics of credence goods. Journal of Economic Literature, $\mathrm{n}^{\circ} 44$, p. 5-42.

Fernandez-Cornejo J. (1996). The microeconomics impact of IPM adoption: theory and application. Agricultural and Resource Economic review, vol. 25, p. 149-160. 
Ferron P. (1999). Protection intégrée des cultures : évolution du concept et de son application. In Fraval A., Silvy C., « $L a$ lutte biologique (II)». Dossiers de l'environnement de l'INRA, n ${ }^{\circ}$ 19, p. 7-18.

Frisvold G. B., Fernicola K., Langworthy M. (2001). Market returns, infrastructure and the supply and demand for extension services. American Journal of Agricultural Economics, vol. 83, $\mathrm{n}^{\circ}$ 3, p. 758-763.

Girardin P. (1992). Agriculture intégrée et recherche : comment lever les barrières ? INRA Mensuel, $\mathrm{n}^{\circ}$ 64, p. 29-32.

Godtland E.-M., Sadoulet E., de Janvry A., Murgai R., Ortiz O. (2004). The Impact of Farmer Field Schools on Knowledge and Productivity: A Study of Potato Farmers in the Peruvian Andes. Economic Development \& Cultural, ${ }^{\circ}$ 53, p. 63-920.

Hanson J.-C., Just R.-E. (2001). The potential for transition to paid extension: some guiding economic principles. American Journal of Agricultural Economics, $\mathrm{n}^{\circ} 83$, p. 777-784.

Ingram J., Morris C. (2007). The knowledge challenge within the transition towards sustainable soil management: an analysis of agricultural advisors in England. Land Use Policy, n $^{\circ}$ 24, p. 100-117.

Jeger M.-J. (1997). Approaches to integrated crop protection in university education and training. Agriculture, Ecosystems \& Environment, vol. 64, ${ }^{\circ} 2$, p. 173-179.

Klerkx L., Jansen J. (2010). Building knowledge systems for sustainable agriculture: supporting private advisors to adequately address sustainable farm management in regular service contacts. International Journal of Agricultural Sustainability, vol. 8(3), p. 148-163.

Labarthe P. (2006). La privatisation du conseil technique agricole en question. Évolutions institutionnelles et performances des services de conseil dans trois pays européens (Allemagne, France, Pays-Bas). Thèse de doctorat en sciences économique. Université de Marne-la-Vallée.
Lamine C., Meynard J-M., Perrot N., Bellon S. (2009). Analyse des formes de transition vers des agricultures plus écologiques : les cas de l'agriculture biologique et de la protection intégrée. Innovations Agronomiques, ${ }^{\circ}$ 4, p. 483-493.

Laurent C., Cerf M., Labarthe P. (2006). Agricultural extension services and market regulation: learning from a comparison of six EU countries. European Journal of Agricultural Education and Extension, vol. 12, $\mathrm{n}^{\circ} 1$, p. 5-16.

Lohr L., Park T., Higley L. (1999). Farmer risk assessment for voluntary insecticide reduction. Ecological Economics, vol. 30, $\mathrm{n}^{\circ} 1$, p. 121-130.

Loyce C., Rolland B., Bernicot M.-H., Bouchard C., Doussinault G., Haslé H., Meynard J.-M. (2001). Les variétés de blé tolérantes aux maladies : une innovation majeure à valoriser par des itinéraires techniques économes. Perspectives Agricoles, $\mathrm{n}^{\circ} 268$, p. 50-56.

Lucas P., Meynard J.-M. (2000). La protection intégrée des cultures à l'INRA. Paris, Rapport à la direction scientifique EFA, $28 \mathrm{p}$.

Meynard J.-M., Savini I. (2003). La désintensification : point de vue d'un agronome. Dossier de l'environnement de l'INRA, $\mathrm{n}^{\circ} 24$, p. 23-33.

Morison M. (2007). Conduite intégrée du colza d'hiver pour une réduction de l'utilisation des pesticides. Rapport de recherche dans le cadre du programme du MEDD 2004-2007 «Évaluation et réduction des risques liés à l'utilisation des pesticides », $49 \mathrm{p}$.

Mundler P., Labarthe P., Laurent C. (2006). Les disparités d'accès au conseil. Le cas de la région Rhône-Alpes. Économie rurale, $n^{\circ} 291$, p. 26-41.

Paillotin G., (2008). Ecophyto 2018. Chantier 15 « agriculture écologique et productive ». Rapport pour le ministère de l'Agriculture et de la Pêche. http:// www.agriculture.gouv.fr/sections/publications/rapports/ecophyto-2018 
RECHERCHES

Philippe AUJAS, Anne LACROIX, Stéphane LEMARIÉ, Raymond REAU

Reau R., Meynard J.-M., Robert D., Gitton C. Ricker-Gilbert J., Norton G.-W., Alwang J., (1996). Des essais factoriels aux essais Miah M., Feder G. (2008). Cost-EffectiConduite de culture. Expérimenter sur les veness of Alternative Integrated Pest Manaconduites de cultures. Comité Potentia- gement Extension Methods: An Example lités-ACTA, p. 52-62.

from Bangladesh. Review of Agricultural Economics, n $^{\circ} 30$, p. 252-269.

Rémy J. (2006). Portrait social des conseillers : retour sur image. In Rémy J., Brives H., Lémery B. (éds). Co-édition Educagri-INRA, " Conseiller en agriculture », p. 83-99.

Viaux P., Le Monnier de Gouville E. (1994). Le temps consacré par les agriculteurs à la prise de décision et au suivi technique des cultures. Institut Supérieur Agricole de Beauvais, $91 \mathrm{p}$. 